\title{
FlexibleUreteroscopy and Holmium:YAG Laser Lithotripsy for Stone Disease in Patients with Bleeding Diathesis: a Systematic Review of the Literature
}

\author{
Omar M Aboumarzouk, Bhaskar K. Somani, Manoj Monga \\ Department of Urology, Wales Deanery, Cardiff, Wales (OMA), University Hospitals Southampton NHS \\ Trust, Southampton (BKS), United Kingdom and Glickman Urological \& Kidney Institute, Cleveland \\ Clinic, Department of Urology (MM), Cleveland, Ohio, USA
}

\section{ABSTRACT}

Introduction and Objectives: The management of urolithiasis in patients on anticoagulants presents a challenge to the endourologist. Due to multiple comorbidities, it may be impossible to safely discontinue the anticoagulant treatment. Other modalities such as shock wave lithotripsy and PCNL are contraindicated in these patients, so ureteroscopic treatment may be the only option. We conducted a systematic review of the literature to look at the safety and efficacy of ureteroscopic management in these patients.

Methods: Systematic review and quantitative meta-analysis was performed using studies identified by a systematic electronic literature search from January 1990 to August 2011. All articles reporting on treatment for stones in patients with a bleeding diathesis using ureteroscopy and a Holmium:YAG laser were included. Two reviewers independently extracted the data from each study. The data was included into a meta-analysis and discussed.

Results: Three studies were identified reporting on 70 patients (73 procedures). All patients had stone fragmentation using Holmium laser. The mean stone size was $13.2 \mathrm{~mm}$ with a range of $5-35 \mathrm{~mm}$. The quality of the included studies was modest. Stone free status was achieved in sixty-four patients (87.7\%). There were no major complications and only $11 \%$ of the patients developed minor complications with only $4 \%$ rate of minor bleeding.

Conclusions: Retrograde stone treatment using ureteroscopy and holmium laser lithotripsy can be safely performed in patients with bleeding diathesis with a low complication rate.

\section{ARTICLE INFO}

\section{Key words:}

Ureteroscopy; Laser Therapy; Lithotripsy; Bleeding time; Blood Coagulation Disorders; Urinary Calculi

Int Braz J Urol. 2012; 38: 298-306

Submitted for publication: October 13, 2011

Accepted after revision: November 29, 2011

\section{INTRODUCTION}

Nephrolithiasis is a common condition affecting the population with a peak incidence around the third to fourth decade of life (1). The lifetime risk of urolithiasis in the general population is $13 \%$ in men and $7 \%$ in women (2).

The preferred treatment modalities for ureteric calculi include shock wave lithotripsy
(SWL) or ureteroscopy (URS) $(3,4)$. With the advancement in technology of fibre optics and the production of smaller calibre ureteroscopes, ureteroscopic extraction has led to a higher stone free rate than SWL and is recommended as first line management for ureteric calculi (5-8).

However, despite the advancements made in the instrumentation, urologists have always opted to correct coagulopathy before undertak- 
ing endourological procedures (7). This poses a controversial question concerning the management of patients who are anticoagulated or have a coagulopathy (9). SWL and percutaneous nephrolithotomy are contraindicated in these patients and correction of coagulopathy is recommended before endoscopic procedures $(9,10)$. However, despite the use of low molecular weight heparin for thromboembolic protection, patients can still develop organ or life threatening clots (10). Conversely, if coagulopathy was not reversed, the procedures run the risk of causing continual bleeding or haematoma formation (10).

In view of all these facts, we aimed to conduct a systematic review to assess the safety and efficacy of ureteroscopic procedures in patients with bleeding diathesis.

\section{MATERIALS AND METHODS}

\section{Search strategy and study selection}

The systematic review was performed according to the Cochrane diagnostic accuracy reviews guidelines. The search strategy was conducted to find relevant studies from MEDLINE (1990- March 2011), EMBASE (1990- March 2011), Cochrane Central Register of Controlled Trials CENTRAL (in The Cochrane Library - Issue 1, 2011), CINAHL (1990- March 2011), Clinicaltrials.gov, Google Scholar and Individual urological journals.

Terms used included: 'ureteroscopy', 'coagulopathy', 'anticoagulant', 'warfarin', 'bleeding', 'urolithiasis', 'aspirin', 'coumarin', 'clopidogrel', 'thrombocytopenia', and 'calculi.

Mesh phrases included: ("Ureteroscopy"[Mesh]) AND "Blood Coagulation Disorders"[Mesh], ("Anticoagulants"[Mesh]) AND "Ureteroscopy”[Mesh], ("Ureteroscopy”[Mesh]) AND "Hemorrhage"[Mesh], ("Anticoagulants"[Mesh]) AND ( "Lasers"[Mesh] OR "Laser Therapy”[Mesh] ), ("Lasers”[Mesh]) AND “Calculi”[Mesh]) AND “Anticoagulants”[Mesh], ("Anticoagulants"[Mesh]) AND "Calculi"[Mesh], ("Ureteroscopy”[Mesh]) AND “Aspirin”[Mesh], ("Ureteroscopy”[Mesh]) AND "clopidogrel” [Supplementary Concept], ("Ureteroscopy”[Mesh]) AND "Coumarins"[Mesh], and ("Ureteroscopy”[Mesh]) AND "Thrombocytopenia"[Mesh], ("Kidney Calculi”[Mesh] OR "Ureteral Calculi”[Mesh]) AND
“Aspirin”[Mesh], (“Coumarins”[Mesh]) AND ( "Kidney Calculi”[Mesh] OR "Ureteral Calculi”[Mesh] ), ("Kidney Calculi”[Mesh] AND "Ureteral Calculi”[Mesh]) AND "Coumarins"[Mesh], and ("Thrombocytopenia”[Mesh]) AND ( "Kidney Calculi”[Mesh] OR "Ureteral Calculi”[Mesh]).

Reference lists of previous reviews and previous trials were included; papers in languages other than English were included, references of searched papers were evaluated for potential inclusion, and recently published versions were included if the publication was duplicated. Authors of the included studies were contacted whenever the data was not available or not clear.

Two reviewers (OA and BS) identified all studies that appeared to fit the inclusion criteria for full review. Each reviewer independently selected studies for inclusion in the review. Disagreement between the two extracting authors was resolved by consensus. If consensus between the two reviewers could not be reached, a third author (MM) was deferred to for arbitration and consensus.

\section{Data extraction and analysis}

Studies reporting on the treatment of patients with a bleeding diathesis with flexible ureteroscopy and laser lithotripsy were included. Patients included were adults with a bleeding diathesis who had urinary stones. The following variables were extracted from each study: period of the study; country of origin of the study; study population demographics; type of anticoagulant used or coagulopathy; stent insertion; stone free rates; follow up; and complications. The data of each study was grouped into a meta-analysis to allow a numerical representation of the results. A quality assessment of harms using the McHarm scale was conducted for each included study (11). We used Review Manager (RevMan 5.0.23) to plot the quality assessment of harms tables.

\section{RESULTS}

The literature search yielded 199 studies, of which 165 were excluded by title or abstract for non-relevance to the aims of this review or not reporting on ureteroscopy treatment of patients with a bleeding diathesis (Figure-1). Eight 
Figure 1 - Flowchart for article selection process of the review.

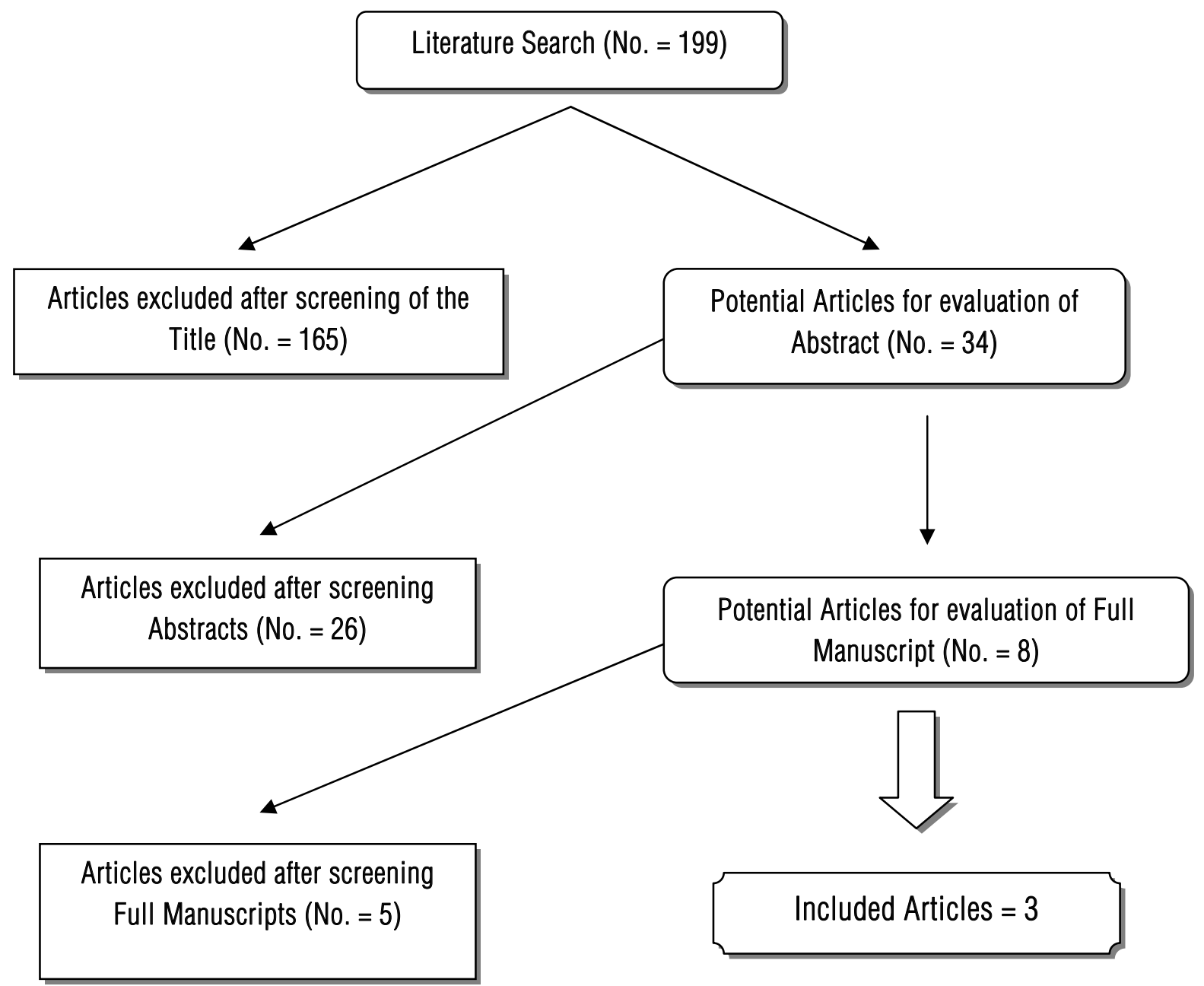

studies were then retrieved for further assessment, of which three were included in the review $(7,8,12)$. All the included studies were published between 1998 and as recent as 2008, reflecting the continued debate of how to treat stones in patients with bleeding diathesis.

All 3 studies were retrospective studies; however, Turna et al. also compared the anticoagulated group to a similar group of patients as a control group. All the studies reported on the variables indicated in the 'data extraction section' and were plotted into Table-1. Wherever data was not available in the reports or there was not enough clarification, lead authors were contacted to get the raw data.

Five articles were excluded after reading the full manuscript. One study was not included since the authors looked at all treatment modalities for urinary stones and though mentioned that 8 patients were ureteroscopically treated only 2 were holmium laser treated (10). Furthermore, the authors had not provided demographic, coagulopathy, or stone details separately for these patients and therefore could not be extracted. Attempts at contacting the author were unsuccessful. The remaining four studies did not look 


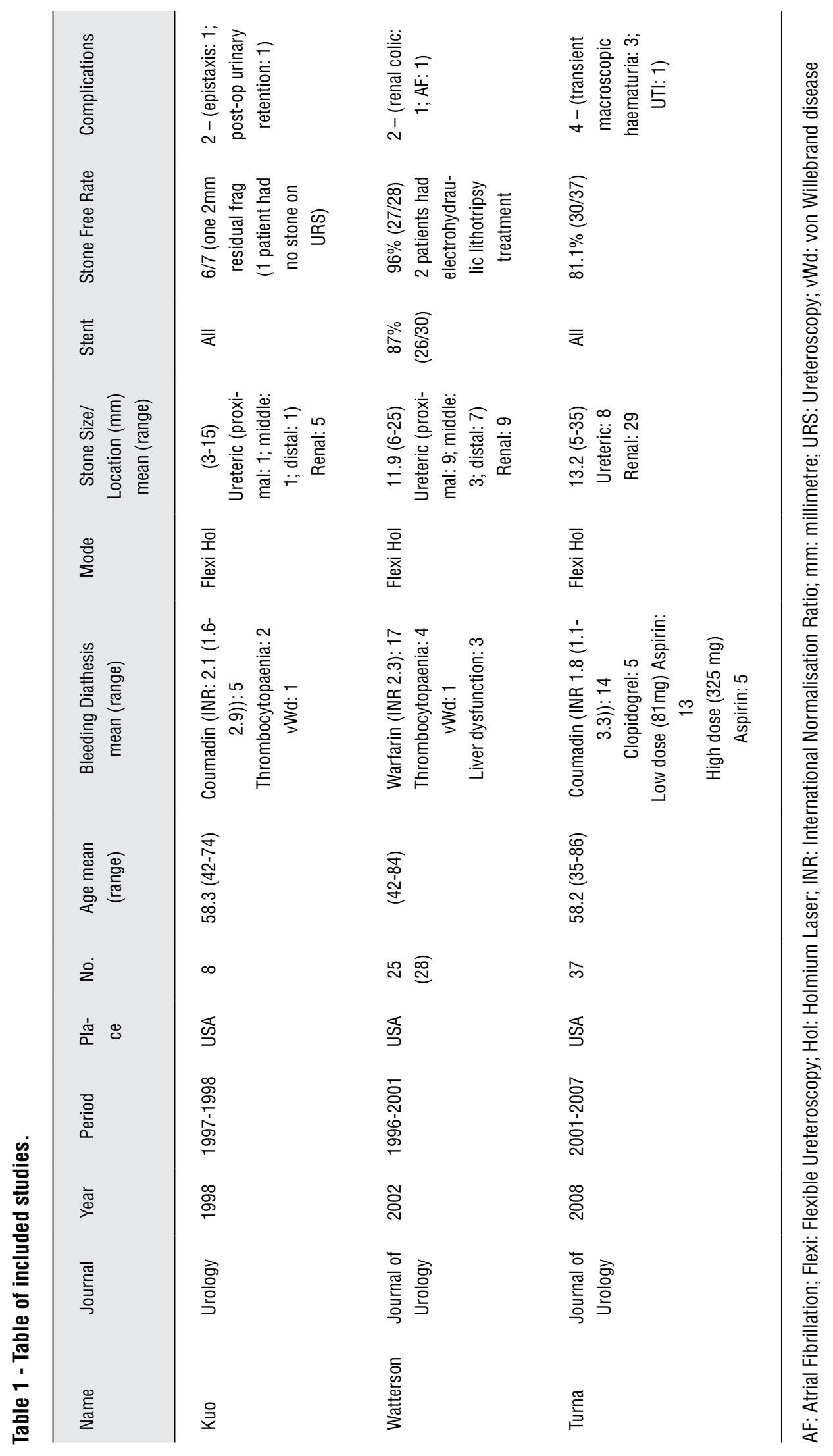


at patients with bleeding diathesis and therefore were excluded $(2,4,9,13)$.

\section{Characteristics of the included studies}

All the studies were conducted in the USA (Table-1). Seventy patients who underwent 73 procedures were included in this review. The study population was composed of patients with 35 to 86 years old. All patients had some sort of coagulopathy including 36 patients on warfarin, 6 patients with thrombocytopaenia, 2 with von Willebrand disease, 3 had liver dysfunction, 5 on clopidogrel, 13 on low dose aspirin, and 5 patients on a high dose aspirin. None of the patients had their coagulopathy reversed, except for 2 patients who had thrombocytopaenia and had recently had chemotherapy; both were given 2 units of platelets for fear of the platelet count dropping further $(7,12)$. The mean international normalization ratio (INR) for the patients on warfarin was 2.1 with a range of 1.1-3.3. Turna et al. had included patients on coumadin; however, their INR was 1.1, and there was no mention of how many patients with subtherapeutic INR levels were included. All patients were treated with a flexible ureteroscopy and a holmium:YAG laser. The stone sizes ranged from 3-35mm with 43 renal stones and 30 ureteric, of which 10 were proximal, 4 middle and 8 distal ureteric. Turna et al. had not mentioned the location of the ureteric stones.

Two studies routinely stented their patients after ureteroscopy and holmium:YAG laser fragmentation. However, the third study (by Watterson et al.) did not differentiate between the patients who had holmium treatment and those that had electrohydraulic lithotripsy and stent insertion, therefore their data was not included.

With regards to stone free rate, $87.7 \%$ (64/73) of the patients were stone free. In this review, none of the patients developed any major complications and 11\% (8/73) of the patients developed minor complications; however, five of the patients had complications unrelated to their coagulopathy. This brought the complication rate that could be attributed to an anticoagulated state, i.e. bleeding, to $4.1 \%$ (3/73). These three patients developed transient macroscopic haematuria for at least 3 days but did not require continuous blad- der irrigations, secondary procedures or blood transfusions (8). The five other complications included one patient who developed a post-operative urinary retention, one patient developed worsening renal colic attributed to stone passage, another developed atrial fibrillation, another developed a urinary tract infection and the last developed an epistaxis. The epistaxis was attributed to ketorolac; however, there was no mention of how they were certain that ketorolac was the cause rather than the coagulopathy. All patients were routinely followed up, however each study varied in the length of follow up. Kuo et al. followed up their patients for 4-6 weeks, while Turna et al. followed up for 4 weeks, and Watterson et al. for 1-2 weeks only. All the patients were stone free and complication free after follow up discharge.

Methodological quality assessment of the included studies

Overall, the quality of the reported studies was modest as two of the studies were reported as retrospective while one was unclear; however it seemed to be retrospective from the methodology. All the included studies might have been subjected to bias as their method of recruitment of patients consisted of recruiting patients from databases; this could lead to selection as well as reporting bias. None of the studies were randomized, blinded $(7,12)$, and only one study had a control group (8). However, the study group (coagulopathy patients) was significantly older than the control group, which poses the question to whether or not these groups could be compared. Furthermore, there was no mention on how the control group patients were selected from the authors' database of 692 patients. This again could be construed as selection bias.

The quality assessment of harms indicates that the studies generally have a low risk of bias concerning reporting the harms that could potentially be caused by the procedure (Figures 2 and 3).

\section{DISCUSSION}

Normalizing coagulopathy pre-operatively is the mainstay of patients' management before surgical procedures. This usually leads to the combined consult and co-ordinated efforts of the sur- 
Figure 2 - Risk of bias graph: review authors' judgements about each risk of bias item presented as percentages across all included studies.

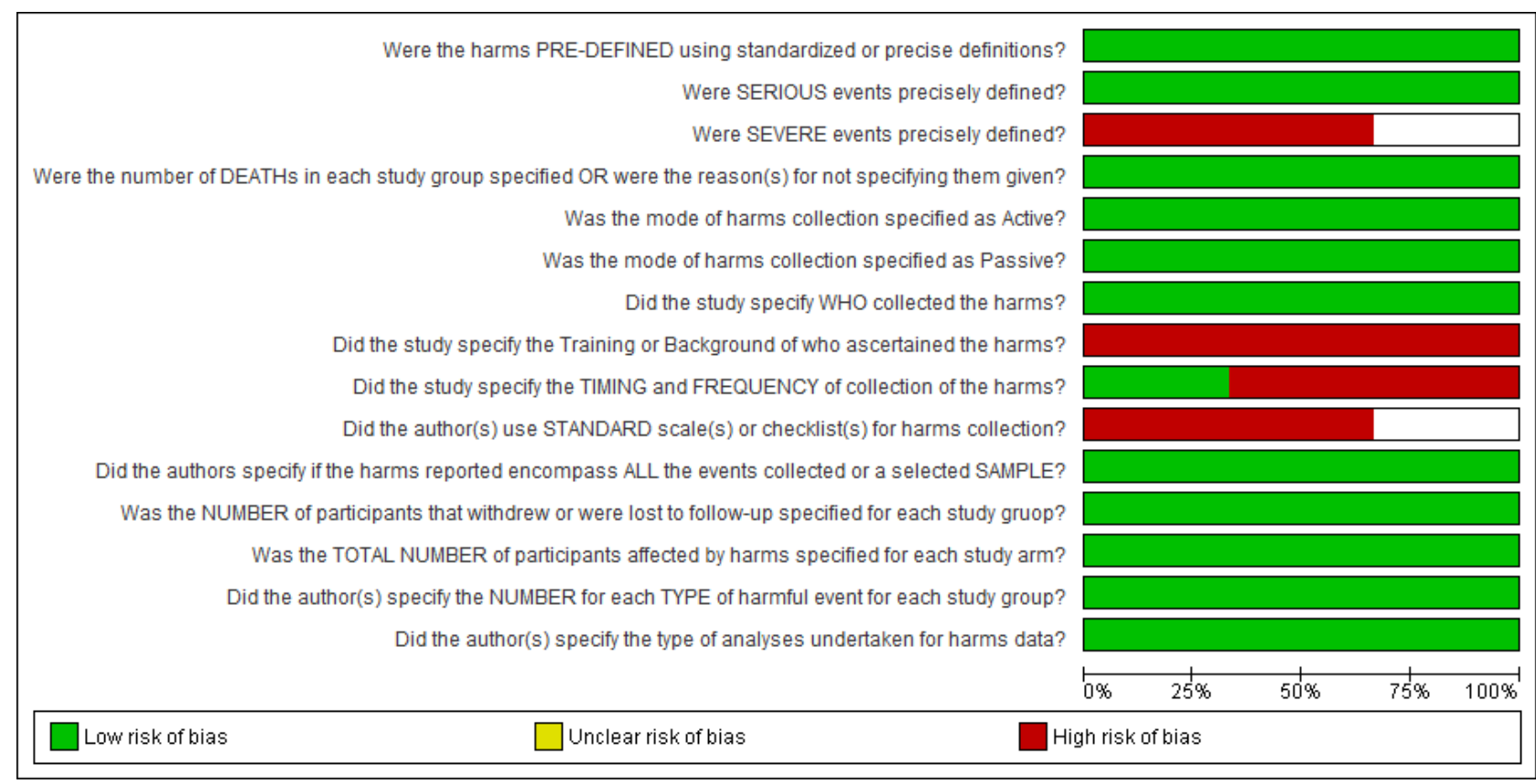

Figure 3 - Risk of bias summary: review authors' judgements about each risk of bias item for each included study.

\begin{tabular}{|c|c|c|c|}
\hline 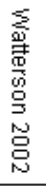 & $\begin{array}{l}\stackrel{-1}{\leftrightarrows} \\
\stackrel{3}{3} \\
\stackrel{N}{\circ} \\
\stackrel{\circ}{\circ}\end{array}$ & $\begin{array}{l}\text { 조 } \\
\text { 口 } \\
\overrightarrow{0} \\
\stackrel{6}{0} \\
\end{array}$ & \\
\hline+ & + & + & Were the harms PRE-DEFINED using standardized or precise definitions? \\
\hline+ & + & + & Were SERIOUS events precisely defined? \\
\hline & & & Were SEVERE events precisely defined? \\
\hline+ & + & + & Were the number of DEATHs in each study group specified OR were the reason(s) for not specifying them given? \\
\hline+ & + & + & Was the mode of harms collection specified as Active? \\
\hline 0 & + & + & Was the mode of harms collection specified as Passive? \\
\hline+ & + & & Did the study specify WHO collected the harms? \\
\hline & & & Did the study specify the Training or Background of who ascertained the harms? \\
\hline & & & Did the study specify the TIMING and FREQUENCY of collection of the harms? \\
\hline & & & Did the author(s) use STANDARD scale(s) or checklist(s) for harms collection? \\
\hline+ & + & + & Did the authors specify if the harms reported encompass ALL the events collected or a selected SAMPLE? \\
\hline+ & + & + & Was the NUMBER of participants that withdrew or were lost to follow-up specified for each study gruop? \\
\hline+ & + & + & Was the TOTAL NUMBER of participants affected by harms specified for each study arm? \\
\hline+ & + & + & Did the author(s) specify the NUMBER for each TYPE of harmful event for each study group? \\
\hline+ & + & + & Did the author(s) specify the type of analyses undertaken for harms data? \\
\hline
\end{tabular}


geons with the haematologists and anaesthetists (12). However, the risk of thromboembolic events during perioperative bridging with heparin is 1-2\% (14). Furthermore, treating the coagulopathy is significantly more costly when compared to patients without coagulopathy undergoing similar procedures (10).

Though other modalities exist for the treatment of large urinary stones, such as SWL, PCNL, and open or laparoscopic surgery, these are contraindicated if the bleeding diathesis is not corrected $(8,10)$. This only leaves ureteroscopic management for these patients (8).

Advancements in endoscope engineering and laser technologies allow an operator to visualise and treat stones in the whole upper urinary system, including the renal calyxes with a reported long-term complication rate of less than $1 \%(12,15,16)$. Holmium lasers provides effective and efficient intracorporeal lithotripsy for even hard stones such as cysteine and calcium oxalate monohydrate stones, and can also be used to ablate upper urinary tract tumours $(7,12)$. Furthermore, holmium lasers offer haemostatic capabilities during the procedure, which gives an additive benefit to patients with bleeding diathesis (12). Lastly, holmium laser energy is rapidly absorbed by water, leading to a minimal risk of ureteric injury if the laser fibre is at least $0.5 \mathrm{~mm}$ away from the ureter and no risk of ureteric perforation if the distance is more than $1 \mathrm{~mm}$ (12).

This review found that the use of flexible ureteroscopes and holmium lasers on patients with bleeding diathesis is not only safe but also efficient, with an overall stone free rate of $87.7 \%$, a minor complication rate of $11 \%$, but only a $4 \%$ rate of minor bleeding, and a major complication rate of $0 \%$.

The validity of the results of systematic review depends on the quality of included studies including selection of participants and inclusion criteria. The studies included seemed to all be retrospective reports of a larger database. Therefore at most this review has a level 2a Levels of Evidence according to CEBM (17). No study evaluated cost analyses.

The other limitation of this review is related to the patient population; the majority of patients were on warfarin. However, the remaining had various other causes for coagulopathy, whether the heterogeneity of the study sample would impact outcomes is not known. However, we aimed at reviewing all patients with coagulopathy and did not target one group. Furthermore, due to the limited number of patients, we did not see a need of conducting sub-groups analysis which would have reduced the cohort even further.

Furthermore, though the level of evidence is considered a $2 \mathrm{a}$, this review has a small cohort of patients (70) from case series basing this evidence on. In addition, no trial or study was found in the literature. This reflects the need for further larger participant studies to further explore the safety and efficacy of ureteroscopy in these patients.

Despite the limitation, grouping of the data was possible and revealed the safety and efficacy of the combined studies. Furthermore, this review opens possibility for further research into the question.

This review has shown that it is not only safe but also efficient to treat patients suffering with urinary stones and afflicted with a bleeding diathesis with ureteroscopy and holmium laser. This can have cost benefits in practice as patients on anticoagulants need not undergo reversal and most patients with coagulopathy need further management to support their coagulation system.

Future research efforts should be concentrated on higher quality, more rigorous evaluation of ureteroscopic treatment in these groups of patients. Studies should be multi-institutional and protocol driven, preferably peer reviewed before the start. Studies should be prospectively evaluated and include a control group of patients who are not anticoagulated for comparison. A detailed evaluation of the different types of bleeding diathesis such as patients on warfarin, clopidogrel, thrombocytopenia or haemophilia should be analyzed individually rather than as a whole. Furthermore, health economic outcome measures should be analyzed.

\section{CONCLUSIONS}

The use of ureteroscopy with the holmium laser is a safe and efficient modality for treating patients with urinary tract calculi who also have a bleeding diathesis or are anticoagulated. Further- 
more, these patients do not need their coagulopathy reversed, which leads to reduction the risk of thromboembolism with very minimal short-term complications and no long term consequence.

\section{CONFLICT OF INTEREST}

None declared.

\section{REFERENCES}

1. Ramello A, Vitale C, Marangella M: Epidemiology of nephrolithiasis. J Nephrol. 2000; 13(Suppl 3): S45-50.

2. Argyropoulos AN, Tolley DA: SWL is more cost-effective than ureteroscopy and Holmium:YAG laser lithotripsy for ureteric stones: A comparative analysis for a tertiary referral centre. British Journal of Medical \& Surgical Urology. 2010; 3: 65-71.

3. Hendrikx AJ, Strijbos WE, de Knijff DW, Kums JJ, Doesburg WH, Lemmens WA: Treatment for extended-mid and distal ureteral stones: SWL or ureteroscopy? Results of a multicenter study. J Endourol. 1999; 13: 727-33.

4. Verze P, Imbimbo C, Cancelmo G, Creta M, Palmieri A, Mangiapia $F$, et al.: Extracorporeal shockwave lithotripsy vs ureteroscopy as first-line therapy for patients with single, distal ureteric stones: a prospective randomized study. BJU Int. 2010; 106: 1748-52.

5. Marchant F, Storme 0, Osorio F, Benavides J, Palma C, Ossandón E: Prospective trial comparing shock wave lithotripsy and ureteroscopy for management of distal ureteral calculi. Actas Urol Esp. 2009; 33: 869-72.

6. Lee YH, Tsai JY, Jiaan BP, Wu T, Yu CC: Prospective randomized trial comparing shock wave lithotripsy and ureteroscopic lithotripsy for management of large upper third ureteral stones. Urology. 2006; 67: 480-4.

7. Kuo RL, Aslan P, Fitzgerald KB, Preminger GM: Use of ureteroscopy and holmium:YAG laser in patients with bleeding diatheses. Urology. 1998; 52: 609-13.
8. Turna B, Stein RJ, Smaldone MC, Santos BR, Kefer JC, Jackman SV, et al.: Safety and efficacy of flexible ureterorenoscopy and holmium:YAG lithotripsy for intrarenal stones in anticoagulated cases. J Urol. 2008; 179: 1415-9.

9. Williams SB: Is continuing warfarin in the perioperative period safe for patients undergoing urologic procedures? Eur Urol. 2011; 59: 372-3.

10. Klingler HC, Kramer G, Lodde M, Dorfinger K, Hofbauer J, Marberger M: Stone treatment and coagulopathy. Eur Urol. 2003; 43:75-9.

11. Santaguida P, Raina P, Ismaila A: McMaster Quality Assessment Scale of Harms (McHarm) for primary studies: Manual for use of the McHarm. Available from: http://hiru. mcmaster.ca/epc/mcharm.pdf

12. Watterson JD, Girvan AR, Cook AJ, Beiko DT, Nott L, Auge BK, et al.: Safety and efficacy of holmium: YAG laser lithotripsy in patients with bleeding diatheses. J Urol. 2002; 168: 442-5.

13. Sofer M, Watterson JD, Wollin TA, Nott L, Razvi H, Denstedt JD: Holmium:YAG laser lithotripsy for upper urinary tract calculi in 598 patients. J Urol. 2002; 167: 31-4.

14. Kaatz S, Paje D: Update in bridging anticoagulation. J Thromb Thrombolysis. 2011; 31: 259-64.

15. Grasso M, Beaghler M, Bagley DH, Strup S: Actively deflectable, flexible cystoscopes: no longer solely a diagnostic instrument. J Endourol. 1993; 7: 527-30.

16. Harmon WJ, Sershon PD, Blute ML, Patterson DE, Segura JW: Ureteroscopy: current practice and long-term complications. J Urol. 1997; 157: 28-32.

17. Jeremy Howick BP, Chris Ball, Dave Sackett, Doug Badenoch, Sharon Straus, Brian Haynes, et al.: Centre for Evidence-Based Medicine. Available from: http://www.cebm. net/index.aspx?0=1025.

\section{Correspondence address:}

Dr. Manoj Monga

Glickman Urological \& Kidney Institute, Cleveland Clinic, Department of Urology, Desk Q10-1, 9500 Euclid Ave, Cleveland, Ohio, USA Fax: +1 216 636-0770 E-mail: endourol@yahoo.com 


\section{EDITORIAL COMMENT}

It has been recently published that urolithiasis is an entity associated with metabolic syndrome, which is characterized by hypertension, obesity, diabetes and abnormal lipid levels (1).

As the world drags its way towards obesity, urologists of all around the globe have noticed that, not only kidney stones have become more frequent, but also those patients who present them have more often other co-morbidities. One particular instance is the drug-induced blood diathesis, which is characterized by the use of "blood thinners" for cardiovascular protection.

These phenomena (obesity, metabolic syndrome, kidney stones, blood thinners) have brought upon the endourologist a current and challenging topic that every kidney stone specialist needs to be up-to-date on: Stone treatment versus bleeding diathesis.

The present study reports on what has been published in the literature that could serve as foundation to our decision making process while counseling a stone patient with any kind of bleeding diathesis. Surprisingly, the authors very well presented the lack of prospective (high evidence levels) studies on this matter; however, based on what has been judiciously selected in the literature, stone free and complication rates

\section{REFERENCE}

1. Lange JN, Mufarrij PW, Wood KD, Holmes RP, Assimos DG: The association of cardiovascular disease and metabolic syndrome with nephrolithiasis. Curr Opin Urol. 2012; 22: 154-9. of flexible ureteroscopy with holmium: YAG laser lithotripsy for patients with blood diathesis are similar to healthy individuals.

It is important to emphasize that if one considers doing a retrograde endoscopic stone treatment in a patient with bleeding diathesis, it is strongly advised, based on evidence level 2a, following analogous surgical technique to what has been described in the selected studies of this systematic review:

A) Energy/lithotripsy - there is no scientific support for using other energy than holmium:YAG laser;

B) Scopes - flexible ureteroscopes were used in all cases;

C) Double J - stenting seems to be a wise routine.

D) General anesthesia might be safer (routine in USA), given the obvious risks of spinal puncture in such patients.

In conclusion, due to a pandemy of obesity and its metabolic consequences, kidney stone patients will more often present co-morbidities and also some kind of bleeding diathesis (aspirin, warfarin, clopidogrel), thus, they must be informed that flexible ureteroscopy and holmium:YAG laser lithotripsy is safe and efficient for treating their ureteral/renal stones.

Dr. Renato Nardi Pedro
Emphasis on Kidney stone Treatment
Clinica Padre Almeida, Campinas
SWL Center Coordinator
Ambulatory Surgical Unit Santa Barbara D’Oeste/
UNICAMP
E-mail: rnpedro@gmail.com

Dr. Renato Nardi Pedro adre Almeida, Campinas UNICAMP

E-mail: rnpedro@gmail.com 